ORIGINAL BREVE

\title{
Relación entre circunferencia de la pantorrilla y velocidad de la marcha en pacientes adultos mayores en Lima, Perú
}

\author{
Gregory Mishell Díaz Villegas ${ }^{a, *}$ y Fernando Runzer Colmenares ${ }^{b}$ \\ a Servicio de Geriatría, Centro Médico Naval, Lima, Perú \\ b Servicio de Geriatría, Centro Médico Naval, Centro de Investigación del Envejecimiento (CIEN), Facultad de Medicina Humana, Universidad de San Martin de Porres, Lima, Perú
}

\section{INFORMACIÓN DEL ARTÍCULO}

\section{Historia del artículo:}

Recibido el 8 de mayo de 2014

Aceptado el 3 de junio de 2014

On-line el 28 de julio de 2014

\section{Palabras clave:}

Adultos mayores

Circunferencia de la pantorrilla

Velocidad de la marcha

\begin{abstract}
R E S U M E N
Objetivo: Conocer la asociación entre la circunferencia de pantorrilla y velocidad de la marcha en pacientes adultos mayores del Servicio de Geriatría del Centro Médico Naval.

Material y métodos: Estudio de tipo descriptivo, transversal y de correlación. Se midió el perímetro de mayor circunferencia de la pantorrilla en la pierna izquierda (pierna derecha de las personas zurdas) y la velocidad de la marcha en pacientes mayores de 65 años. Se realizó un análisis bivariado para evaluar la asociación entre la circunferencia de la pantorrilla y la velocidad de la marcha construyendo un modelo de regresión logística sobre la velocidad de la marcha ajustado por circunferencia de pantorrilla, estado funcional y edad.

Resultados: El promedio de edad fue de 79,37 $\pm 8,71$ años. El 59,71\% perteneció al sexo masculino, el 30,97\% presentó una marcha de velocidad lenta y la media de la circunferencia de pantorrilla fue $33,42 \pm 5,61 \mathrm{~cm}$. En el análisis bivariado, la circunferencia de la pantorrilla tuvo una media de $30,35 \pm 3,74 \mathrm{~cm}$ en los participantes con velocidad de la marcha lenta $(<0,8 \mathrm{~m} / \mathrm{seg})$, significativamente menor al grupo de marcha normal que obtuvo una media de 33,51 $\pm 3,26 \mathrm{~cm}$. Mediante un modelo de regresión logística para evaluar las variables asociados con velocidad de la marcha lenta, la circunferencia de la pantorrilla, el estado funcional y la edad presentaron asociación estadísticamente significativa. Conclusiones: A menor circunferencia de la pantorrilla, encontramos disminución de la velocidad de la marcha en la población mayor de 65 años.
\end{abstract}

(C) 2014 Publicado por Elsevier España, S.L.U. en nombre de SEGG.

\section{Calf circumference and its association with gait speed in elderly participants at Peruvian Naval Medical Center}

\section{A B S T R A C T}

Objective: To evaluate the association between calf circumference and gait speed in elderly patients 65 years or older at Geriatric day clinic at Peruvian Centro Médico Naval.

Material and methods: Cross-sectional, retrospective study. We assessed 139 participants, 65 years or older at Peruvian Centro Médico Naval including calf circumference, gait speed and Short Physical Performance Battery. With bivariate analyses and logistic regression model we search for association between variables.

Results: The age mean was 79.37 years old (SD: 8.71 ). $59.71 \%$ were male, the $30.97 \%$ had a slow walking speed and the mean calf circumference was $33.42 \mathrm{~cm}$ (SD: 5.61). After a bivariate analysis, we found a calf circumference mean of $30.35 \mathrm{~cm}$ (SD: 3.74 ) in the slow speed group and, in normal gait group, a mean of $33.51 \mathrm{~cm}$ (SD: 3.26 ) with significantly differences. We used logistic regression to analyze association with slow gait speed, founding statistically significant results adjusting model by disability and age. Conclusion: Low calf circumference is associated with slow speed walk in population over 65 years old.

\footnotetext{
* Autor para correspondencia.

Correo electrónico: gregorymishell@hotmail.com (G.M. Díaz Villegas).
} 


\section{Introducción}

El proceso de envejecimiento está relacionado con cambios significativos en la composición corporal, con una disminución de la masa corporal magra y con un aumento de la masa grasa visceral ${ }^{1}$. La pérdida de masa muscular se asocia con bajo rendimiento físico, inactividad física, velocidad de andar lento y disminución de la movilidad, y estos factores se reconocen como características comunes del síndrome de fragilidad. De acuerdo con Fried et al., la pérdida de la masa corporal magra es muy importante en la progresión del estado de fragilidad de los sujetos adultos mayores ${ }^{3}$.

La pérdida de masa muscular relacionada con la edad también se asocia con un mayor riesgo de discapacidad y todas las causas de mortalidad en la población anciana. La absorciometría de energía dual de rayos X (DEXA por su siglas en inglés Dual-energy X-ray absorptiometry) y el análisis de impedancia bioeléctrica (BIA por sus siglas en inglés Bioelectrical impedance analysis) son métodos para evaluar la masa corporal magra; sin embargo, no son de fácil acceso. Las mediciones antropométricas podrían ser muy útiles para la evaluación clínica del estado nutricional, masa muscular y sarcopenia en los adultos mayores ${ }^{4}$. La circunferencia de la pantorrilla $(\mathrm{CP})$ es una técnica de bajo costo y tiene correlación positiva con la masa muscular, una medida menor a $31 \mathrm{~cm}$ se ha asociado a discapacidad $^{5}$.

La velocidad de marcha es una prueba sencilla de realizar y su utilidad para identificar pacientes adultos mayores frágiles ha sido ampliamente demostrada ${ }^{6-8}$. Buchner et al. fueron los primeros en observar una relación entre la fuerza de las piernas y la velocidad de la marcha habitual; esta relación explicaba cómo pequeños cambios en la capacidad fisiológica pueden tener efectos importantes sobre el rendimiento en adultos frágiles, por lo tanto la velocidad de la marcha es una medida útil de rendimiento físico ${ }^{5-9}$.

A pesar de los avances y crecimiento de la investigación, la información sobre cómo los datos antropométricos, el rendimiento físico y la debilidad pueden estar relacionados entre sí aun es incompleta. El objetivo de este estudio es conocer la asociación entre la velocidad de la marcha y la $\mathrm{CP}$.

\section{Material y métodos}

El estudio fue de tipo descriptivo, transversal y de correlación, en la unidad de Clínica de Día del Servicio de Geriatría del Centro Médico Naval «Cirujano Mayor Santiago Távara», durante el año 2013, con una muestra por conveniencia de 139 pacientes. Se basó en la medición directa de la $\mathrm{CP}$, velocidad de la marcha, prueba corta de rendimiento físico (SPPB por sus siglas en inglés), estado funcional y fuerza de prensión en los pacientes mayores de 65 años. Se recolectaron datos sociodemográficos de las historias clínicas, el número de hospitalizaciones y caídas en el último año. Los criterios de inclusión fueron: edad mayor a 65 años y que desearan participar en el estudio previo consentimiento informado. El criterio de exclusión fue: presentar signos de edemas periféricos.

Se realizó la medición directa del mayor perímetro gemelar (pantorrilla) en centímetros, con una cinta de plástico no elástica y flexible, en la pierna izquierda (derecha de las personas zurdas) en posición sentada, con la rodilla y el tobillo en ángulo recto y los pies apoyados en el suelo. Un punto de corte menor de $31 \mathrm{~cm}$ se consideró masa muscular disminuida ${ }^{10,11}$. Se midió el tiempo de la persona al caminar 4 metros y se obtuvo la velocidad de la marcha ( $\mathrm{m} / \mathrm{seg}$ ). Se consideró velocidad lenta la marcha con un punto de corte de $<0,8 \mathrm{~m} / \mathrm{seg}^{9}$.

Se realizó la SPPB que se compone de 3 pruebas cronometradas $^{12}$ : la primera prueba es la evaluación del balance y equilibrio (posición paralela con los pies juntos, posición semitándem y tándem durante 10 segundos), la segunda prueba es la medición de la velocidad de la marcha y la tercera prueba es levantarse de la silla. La puntuación oscila entre 0-12 puntos (mayor puntuación mejor funcionamiento físico). Se midió la fuerza de prensión con el dinamómetro de marca CAMRY modelo EH101-67, solicitando al paciente, en posición sentado, empuñar el dinamómetro con el brazo dominante en ángulo de $90^{\circ}$ y realizar la prensión, Se clasificó baja fuerza muscular como empuñadura menos de 30 y $20 \mathrm{~kg}$ en hombres y mujeres, respectivamente ${ }^{13}$.

Se utilizó la escala de Barthel ${ }^{14}$ para identificar el estado funcional de las actividades básicas de la vida diaria y la escala de Lawton y Brody ${ }^{15}$ para las actividades instrumentales de la vida diaria. Se obtuvieron medidas antropométricas como el peso, la talla y el cálculo del índice de masa corporal (IMC) (peso/talla ${ }^{2}$ ).

Análisis estadístico: se analizó la relación entre variables categóricas mediante prueba de chi-cuadrado y de variables numéricas con prueba de t de Student y prueba de U Mann Whitney según normalidad de variables numéricas. Finalmente se realizó un modelo de regresión logística de variables asociadas con velocidad de la marcha disminuida, inicialmente bivariante y posteriormente ajustando la CP por edad y situación funcional, calculándose el valor de esta asociación en odds ratio (OR) con su intervalo de confianza al 95\% (IC 95\%). Se utilizó el programa estadístico Stata V.12.1.

\section{Resultados}

El promedio de edad fue de 79,37 $\pm 8,71$ años, el 59,71\% ( $n=83$ ) pertenecía al sexo masculino y el $72,82 \%(n=75)$ eran casados. Se encontró una media de la CP de 33,42 $\pm 5,61 \mathrm{~cm}$, el 30,97\% presentó una velocidad de la marcha lenta. Los scores de las escalas de Barthel y Lawton tuvieron una media de 78,15 $\pm 25,46$ y 3,6 $\pm 3,11$ respectivamente. El promedio de hospitalizaciones durante el año fue $0,41 \pm 0,64$ y de caídas $1,02 \pm 1,74$. El score de la prueba corta de rendimiento físico (SPPB) presentó una media de $6,68 \pm 3,74$ y la fuerza de prensión tuvo una media de $19,69 \pm 9,87 \mathrm{~kg}$. El 40,46\% presentó baja de peso y el IMC presentó una media de 25,17 $\pm 4,28$.

En el análisis bivariado, la edad promedio en el grupo con velocidad de la marcha lenta fue de $80,38 \pm 8,7$ años y 78,36 $\pm 8,36$ años en la población con marcha normal. La CP tuvo una media de $30,35 \pm 3,74 \mathrm{~cm}$ en los participantes con velocidad de la marcha lenta, menor al grupo de marcha normal que presentó una media de $33,51 \pm 3,26 \mathrm{~cm}(\mathrm{p}<0,05)$. La escala de Barthel, la escala de Lawton, el SPPB y la fuerza de prensión presentaron promedios significativamente menores en los pacientes con velocidad de la marcha lenta comparados con los participantes que presentaron una velocidad de marcha normal $(p<0,05)$. Se encontró un promedio mayor de hospitalizaciones en los pacientes con velocidad de marcha lenta $(\mathrm{p}<0,05)$, mientras que las caídas, IMC y auto reporte de baja de peso no presentaron mayor implicancia estadística (tabla 1 ).

Mediante un modelo de regresión logística para evaluar las variables asociados con velocidad de la marcha lenta, la CP, el estado funcional y la edad encontramos que la CP presenta un OR ajustado de 1,79 (IC 95\% 1,61-1,89), la escala de Barthel un OR 0,92 (IC $95 \% 0,88-0,97$ ) y la edad un OR 1,12 (IC 95\% 1,04-1,9) presentando asociación estadísticamente significativa ( $\mathrm{p}<0,05)$. (tabla 2 ).

\section{Discusión}

En el presente estudio se encontró que mientras la CP disminuye, la velocidad de la marcha enlentece, resultados similares fueron encontrados por Landi et al., en una población basada en el estudio Invecchiamento e Longevità nel Sirente (ilSIRENTE), donde tuvo por objetivo establecer la relación entre la CP con fragilidad, rendimiento físico, fuerza muscular y estado funcional en una población mayor de 80 años. Encuentran que la velocidad de la marcha, medida en 4 metros, mejora conforme aumenta la $\mathrm{CP}$, 
Tabla 1

Características y análisis bivariado del estudio circunferencia de la pantorrilla y velocidad de la marcha en pacientes mayores de 65 años

\begin{tabular}{|c|c|c|c|c|}
\hline \multirow[t]{2}{*}{ Variable } & \multirow[t]{2}{*}{ Media \pm DE o \% (total $=139)$} & \multicolumn{2}{|c|}{ Velocidad de la marcha } & \multirow[t]{2}{*}{$\mathrm{P}^{*}$} \\
\hline & & $\begin{array}{l}\text { Lenta }(<0,8 \mathrm{~m} / \mathrm{seg}) \\
\mathrm{n}=35(30,97 \%)\end{array}$ & $\begin{array}{l}\text { Normal } \\
n=78(69,03 \%)\end{array}$ & \\
\hline Edad & $79,37 \pm 8,71$ & $80,38 \pm 8,7$ & $78,36 \pm 8,36$ & $0,87^{a}$ \\
\hline $\begin{array}{l}\text { Sexo } \\
\text { Femenino } \\
\text { Masculino }\end{array}$ & $\begin{array}{l}56(40,29 \%) \\
83(59,71 \%)\end{array}$ & $\begin{array}{l}16(45,71 \%) \\
19(54,29 \%)\end{array}$ & $\begin{array}{l}28(36,36 \%) \\
49(63,64 \%)\end{array}$ & $0,35^{\mathrm{a}}$ \\
\hline $\begin{array}{l}\text { Estado civil } \\
\text { Casado/a } \\
\text { Soltero/a } \\
\text { Viudo/a o divorciado/a }\end{array}$ & $\begin{array}{l}75(72,82 \%) \\
4(3,88 \%) \\
24(23,3 \%)\end{array}$ & $\begin{array}{l}19(67,86 \%) \\
0(0 \%) \\
9(32,14 \%)\end{array}$ & $\begin{array}{l}3(4,92 \%) \\
9(14,75 \%)\end{array}$ & $\begin{array}{c}0,09^{\mathrm{b}} \\
49(80,33 \%)\end{array}$ \\
\hline Circunferencia de la pantorrilla en $\mathrm{cm}$ & $33,42 \pm 5,61$ & $30,35 \pm 3,74$ & $33,51 \pm 3,26$ & $0,004^{c}$ \\
\hline Puntaje de Índice de Barthel & $78,15 \pm 25,46$ & $55,57 \pm 28,64$ & $90,8 \pm 11,99$ & $0,00001^{\mathrm{c}}$ \\
\hline Puntaje de escala de Lawton & $3,6 \pm 3,11$ & $1,33 \pm 2,26$ & $4,66 \pm 2,98$ & $0,00001^{\mathrm{c}}$ \\
\hline Puntaje en SPPB & $6,68 \pm 3,74$ & $3,37 \pm 3,14$ & $8,3 \pm 2,93$ & $0,00001^{\mathrm{c}}$ \\
\hline Fuerza de Prensión en $\mathrm{kg}$ & $19,69 \pm 9,87$ & $12,71 \pm 10,62$ & $22,94 \pm 8,07$ & $0,0001^{\mathrm{c}}$ \\
\hline Hospitalizaciones en el último año & $0,41 \pm 0,64$ & $0,6 \pm 0,7$ & $0,26 \pm 0,50$ & $0,006^{c}$ \\
\hline Caídas en el último año & $1,02 \pm 1,74$ & $1,18 \pm 1,76$ & $0,96 \pm 1,75$ & $0,25^{a}$ \\
\hline IMC & $25,17 \pm 4,28$ & $24,14 \pm 4,15$ & $25,65 \pm 4,18$ & $0,07^{c}$ \\
\hline Baja de peso & $53(40,46)$ & $16(50 \%)$ & $23(31,08 \%)$ & $0,06^{\mathrm{b}}$ \\
\hline
\end{tabular}

DE: desviación estándar; IMC: índice de masa corporal; SPPB: short physical performance battery.

a Prueba de U Mann Whitney.

b Prueba de chi².

c Prueba t de Student.

${ }^{*} \mathrm{p}<0,05$.

Tabla 2

Modelo de regresión logística de predicción de velocidad de marcha lenta en pacientes mayores de 65 años ( $\mathrm{n}=139)$

\begin{tabular}{|c|c|c|c|c|}
\hline Variables & OR crudo & $\mathrm{P}^{*}$ & OR ajustado ${ }^{\mathrm{a}}$ & $\mathrm{p}^{*}$ \\
\hline Circunferencia de pantorrilla ${ }^{b}$ & $2,77(1,62-2,96)$ & 0,02 & $1,79(1,61-1,89)$ & 0,05 \\
\hline Independencia funcional (Barthel) & $0,92(0,89-0,95)$ & 0,0001 & $0,92(0,88-0,97)$ & 0,003 \\
\hline Edad en años & $1,03(0,98-1,08)$ & 0,24 & $1,12(1,04-1,9)$ & 0,012 \\
\hline Sexo & & 0,34 & & \\
\hline Masculino & $0,68(0,30-1,53)$ & & & \\
\hline \multicolumn{5}{|l|}{ Grado de instrucción } \\
\hline Secundaria incompleta & $2,58(0,89-7,48)$ & & & \\
\hline Hospitalizaciones & $2,56(1,30-5,13)$ & 0,007 & & \\
\hline Caídas & $1,07(0,85-1,35)$ & 0,54 & & \\
\hline Lawton & $0,64(0,52-0,79)$ & 0,0001 & & \\
\hline SPPB & $0,63(0,53-0,75)$ & 0,0001 & & \\
\hline Fuerza de prensión & $0,87(0,81-0,93)$ & 0,0001 & & \\
\hline IMC & $0,91(0,80-1,04)$ & 0,15 & & \\
\hline Baja de peso & $2,22(0,95-5,19)$ & 0,06 & & \\
\hline
\end{tabular}

IMC: índice de masa corporal; SPPB: short physical performance battery.

a Modelo ajustado por circunferencia de pantorrilla, estado funcional y edad.

b Perímetro de mayor circunferencia de pantorrilla en la pierna izquierda (pierna derecha de las personas zurdas).

* $\mathrm{p}<0,05$.

igualmente mejoría en el rendimiento físico, el estado funcional, la fuerza de prensión y disminución de la gravedad de la fragilidad; sin embargo, luego de ajustar las variables entre el índice de fragilidad, el performance físico y estado funcional en relación con la $\mathrm{CP}$, se observa que la velocidad de la marcha y la funcionalidad pierden significación estadística ${ }^{13}$.

Yves Rolland et al. determinaron que la $\mathrm{CP}$ está relacionada positivamente con la masa músculo esquelética apendicular ${ }^{10} \mathrm{y}$ la pérdida de masa muscular se asocia a peor rendimiento físico, inactividad física, velocidad de andar lento y disminución de la movilidad $^{3}$. Esto es corroborado en nuestro estudio, donde encontramos asociación directa entre la $\mathrm{CP}$, velocidad de marcha lenta y disfuncionalidad.

Nuestros resultados indicaron que los cambios en la CP, el rendimiento físico, el estado funcional y la fuerza de prensión se asocian a la alteración en la velocidad de la marcha. Por lo tanto debemos utilizar esta prueba antropométrica breve, de bajo costo y no invasiva junto con la velocidad de la marcha en la atención primaria de salud con la finalidad de identificar población en riesgo.

\section{Conflicto de intereses}

Los autores declaran no tener ningún conflicto de intereses.

\section{Bibliografía}

1. Thomas DR. Loss of skeletal muscle mass in aging: examining the relationship of starvation, sarcopenia and cachexia. Clin Nutr. 2007;26:389-99.

2. d'Hyver C, Gutiérrez Robledo L. Geriatría. México: Manual Moderno; 2009.

3. Fried LP, Tangen CM, Walston J, Newman AB, Hirsch C, Gottdiener J, et al. Frailty in older adults: Evidence for a phenotype. J Gerontol A Biol Sci Med Sci. 2001;56:146-56.

4. Landi F, Russo A, Liperoti R, Pahor M, Tosato M, Capoluongo E, et al. Midarm muscle circumference, physical performance and mortality: Results from the aging and longevity study in the Sirente geographic area (ilSIRENTE study). Clin Nutr. 2010;29:441-7.

5. Cruz-Jentoft A, Baeyens JP, Bauer JM, Cederholm T, Land F, Martin FC, et al., European Working Group on Sarcopenia in Older People. Sarcopenia: European consensus on definition and diagnosis: Report of the European Working Groupon Sarcopenia in Older People. Age Ageing. 2010:39:412-23.

6. Jürschik Giménez P, Escobar Bravo M, Nuin Orrio C, Botigué Satorra T. Criterios de fragilidad del adulto mayor. Estudio piloto, Aten Primaria. 2011;43:190-6. 
7. Varela L, Ortiz J, Chávez H. Velocidad de la marcha como indicador de fragilidad en adultos mayores de la comunidad en Lima, Perú. Rev Esp Geriatr Gerontol. $2010 ; 45: 22-5$.

8. Runzer Colmenares FM, Samper Ternent R, Al Snih S, Ottenbacher KJ, Parodi JF, Wong R. Prevalence and factors associated with frailty among Peruvian older adults. Arch Gerontol Geriatr. 2014;58:69-73.

9. Osuna Pozoa C, Serra Rexacha J, Viña J, Gómez Cabrerab M, Salvác A, Ruiz D, et al. Prevalencia de sarcopenia en consultas de geriatría y residencias. Estudio ELLI. Rev Esp Geriatr Gerontol. 2013;49:72-6.

10. Rolland Y, Lauwers-Cances V, Cournot M, Nourhashémi F, Reynish W, Rivière D, et al. Sarcopenia, calf circumference, and physical function of elderly women: A cross-sectional study. J Am Geriatr Soc. 2003;51:1120-4.

11. World Health Organization. Physical status: The use and interpretation of anthropometry: Report of WHO Expert Committee. Geneva: WHO; 1995
12. Guralnik JM, Ferrucci L, Pieper CF, Leveille SG, Markides KS, Ostir GV, et al Lower extremity function and subsequent disability: Consistency across studies, predictive models, and value of gait speed alone compared with the Short Physical Performance Battery. J Gerontol A Biol Sci Med Sci. 2000;55: 221-31.

13. Landi F, Onder G, Russo A, Liperoti R, Tosato M, Martone AM, et al. Calf circumference, frailty and physical performance among older adults living in the community. Clini Nutr. 2014;33:539-44

14. Mahoney FI, Barthel DW. Functional evaluation:Tthe Barthel index. Md State Med J. 1965;14:61-5.

15. Lawton MP, Brody EM. Assessment of older people: Self-maintaining and instrumental activities daily living. Gerontologist. 1969;9:179-86. 